\title{
All-Optical Atomic Bose-Einstein Condensates
}

M. D. Barrett, ${ }^{*}$ M.-S. Chang, C. Hamley, K. Fortier, J. A. Sauer, and M. S. Chapman School of Physics, Georgia Institute of Technology, Atlanta, GA

We have created an atomic Bose-Einstein condensate (BEC) using all-optical methods, realizing a long-term objective in the field. Our method is simpler and faster than traditional BEC experiments and offers unique capabilities for atoms and molecules not amenable to magnetic trapping.

\section{Introduction}

Given the tremendous impact of BEC research in last 7 years and the continued growth of the field, it is important to explore different methods for reaching BEC, particularly methods that offer new capabilities, simplicity, or speed. We have recently demonstrated such a method by creating a Bose condensate of ${ }^{87} \mathrm{Rb}$ atoms directly in a crossed-beam optical dipole force trap using tightly focused $\mathrm{CO}_{2}$ gas laser beams [1].

In the broader scope of research with ultracold degenerate gases, our system stands out for several reasons. First, all-optical BEC provides the first new path to achieving BEC since the first pioneering demonstrations [2-4], and it is surprising simple and an order of magnitude faster than standard BEC experiments. Also, optical trapping potentials are essentially spin-independent and hence are well suited for studying the formation and dynamics of spinor condensates. Finally, we can engineer a rich variety of spatial confinements, including large period one- and threedimensional lattices that offer the possibility of optically resolving individual lattice sites.

All-optical methods of reaching the BEC phase transition have been pursued since the early days of laser cooling. Despite many impressive developments beyond the limits set by Doppler cooling, the best previous efforts yielded atomic phase space densities a factor of 3 away from the BEC transition $[5,6]$. Hence, optical traps have played only a supporting role in BEC experiments. The MIT group used a magnetic trap with an 'optical dimple' to reversibly condense a magnetically confined cloud of atoms evaporatively cooled to just above the phase transition [7]. Additionally, Bose condensates created in magnetic traps have been successfully transferred to shallow optical traps for further study [8]. In all these cases, however, magnetic traps provided the principle increase of phase space density (by factors up to $10^{6}$ ) to the BEC transition.

Evaporative cooling in optical traps was first demonstrated in 1994, where, starting with only 5000 atoms, a phase space density increase of a factor of $\sim 30$ was realized [9]. Whereas the first demonstrations of evaporative cooling of alkali atoms in magnetic traps lead quickly to the observation of BEC, the progress in optical traps was slower. A principle challenge faced by all-optical traps is that the small

* present address, Time and Frequency Division, NIST, Boulder, CO 
trap volume provided by the focused lasers (typically much less than $1 \mathrm{~mm}^{3}$ ) limits the number of atoms that can be loaded in the trap. Also, lowering the trap depth to force evaporation also lowers the trap oscillation frequency and the rethermalization rate. Hence the evaporation rate can slow down prohibitively. Finally, optical traps have historically suffered from excessive heating rates either due to technical noise of the trapping laser or the intrinsic spontaneous scattering of photons from the trapping beams $[10,11]$.

Our experiments employ $\mathrm{CO}_{2}$ laser optical traps [12] loaded from a standard ${ }^{87} \mathrm{Rb}$ vapor cell MOT. The MOT is loaded for $5 \mathrm{~s}$ directly from the thermal vapor during which we collect up to $10^{8}$ atoms. After loading the MOT, the cooling configuration is changed to maximize the transfer of atoms to the optical trap. The repump intensity is first lowered to $10 \mu \mathrm{W} / \mathrm{cm}^{2}$ for $20 \mathrm{~ms}$, and then the MOT trap beams are shifted to the red of the trapping transition by $140 \mathrm{MHz}$ for $40 \mathrm{~ms}$. At this point the MOT beams are extinguished and the current in the MOT coils is turned off. In order to optically pump the atoms into the $F=1$ hyperfine states, the repump light is shuttered off $1 \mathrm{~ms}$ before the trap beams are extinguished; we measure the efficiency of the optical pumping to the $F=1$ state to be $>95 \%$. The $\mathrm{CO}_{2}$ laser beams are left on at full power throughout the MOT loading and dipole trap loading process.
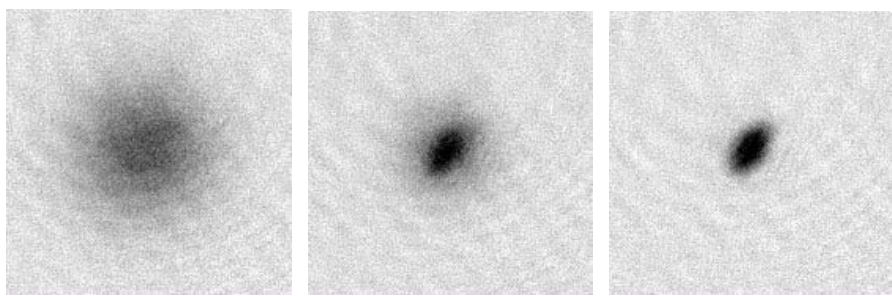

Figure 1: Images of the trapped atoms at different final temperatures following 10 $\mathrm{ms}$ of ballistic expansion to reveal the momentum distribution of the atoms. For the thermal cloud (left) the distribution is isotropic. Appearance of the condensate is revealed by the anisotropic distribution present in the center and right images.

The trapping beams are generated from a commercial $\mathrm{CO}_{2}$ gas laser (Synrad, Model $48-1, \lambda=10.6 \mu \mathrm{m})$. The beams are tightly focused with $f=38 \mathrm{~mm}$ focal length ZeZn aspherical lenses inside the chamber. We have six such lenses inside the chamber forming three orthogonal 1:1 telescopes that allow us to create a wide range of traveling wave and standing wave configurations including a 6 beam 3-D optical lattice. For the condensate work, two crossed traveling waves are used, intersected at right angles; one beam is oriented in the horizontal direction and one beam is inclined at $45^{\circ}$ from the vertical direction. Each beam passes through an acousto-optic modulator to provide independent control of the power in the two beams. Additionally, the beams are frequency shifted $80 \mathrm{MHz}$ relative to each other so that any spatial interference patterns between the two beams are time-averaged to zero [5]. Each beam has a maximum power of $12 \mathrm{~W}$, and the beams are focused to a minimum waist $\sim 50 \mu \mathrm{m}$. In this cross geometry, we initially load $2 \times 10^{6}$ atoms at a 
temperature of $70 \mu \mathrm{K}$ and densities $>10^{14}$ atoms $/ \mathrm{cm}^{3}$. Forced evaporative cooling of the atomic sample is achieved by lowering the trap beam powers 50 -fold over $2 \mathrm{~s}$. The BEC transition occurs at temperature of $\sim 300 \mathrm{nK}$ and beam powers of $300 \mathrm{~mW}$. Our pure condensates contain $3 \times 10^{4}$ atoms. An absorptive image of the condensate is shown in Figure 1.

Our success in achieving BEC in an optical trap hinged on the very high spatial and phase space densities achieved during the loading process. The initial spatial density loaded into the dipole trap exceeded $10^{14}$ atoms $/ \mathrm{cm}^{3}$, which was 3 orders of magnitude higher than the loading MOT and almost an order of magnitude higher than previously achieved by direct loading of an optical trap [13]. The initial phase space density was $>10^{-3}$, which was also 3 orders of magnitude higher than the MOT. Given the well-known density limitations in laser cooling due to reabsorption of scattered radiation and photo-associative losses induced by the cooling fields, it is surprising that such high densities could be achieved in an optical trap using only standard sub-Doppler cooling techniques. Although loading of optical dipole traps has been the subject of several experimental [13,14] and theoretical [15] studies, none of these apply specifically to our configuration. Clearly, there is a need to better understand the loading dynamics of optical dipole traps.

Table 1

\begin{tabular}{l|l|l|l}
\hline \multicolumn{1}{c}{ Single beam } & \multicolumn{1}{l}{ 1-D Lattice } & Cross \\
\hline Trapped atoms & $1 \times 10^{6}$ & $4000 /$ microtrap & $2 \times 10^{6}$ \\
\hline Temperature & $14 \mu \mathrm{K}$ & $35 \mu \mathrm{K}$ & $75 \mu \mathrm{K}$ \\
\hline Mean trap frequency & $145 \mathrm{~Hz}$ & $9000 \mathrm{~Hz}$ & $1500 \mathrm{~Hz}$ \\
\hline Potential depth & $250 \mu \mathrm{K}$ & $450 \mu \mathrm{K}$ & $500 \mu \mathrm{K}$ \\
\hline Spatial density & $>10^{12} \mathrm{~cm}^{-3}$ & $>10^{13} \mathrm{~cm}^{-3}$ & $>10^{14} \mathrm{~cm}^{-3}$ \\
\hline Phase space density & 0.0001 & 0.0005 & $0.001-0.005$ \\
\hline Elastic collision rate & $40 \mathrm{~s}^{-1}$ & $700 \mathrm{~s}^{-1}$ & $10,000 \mathrm{~s}^{-1}$ \\
\hline
\end{tabular}

We have compared the loading of different geometry $\mathrm{CO}_{2}$ laser traps. In addition to the cross geometry, we have studied traps formed with a single focused beam, as well as a 1-D lattice trap obtained by retro-reflecting the laser beam. The latter produces an array of microtraps separated by half the wavelength $(5.3 \mu \mathrm{m})$ [13]. Some important properties of these traps are shown in Table 1. While each of these traps provide much higher spatial and phase space densities than the MOT, only the direct loading provided by the cross trap is sufficient to obtain degeneracy in our system. In the case of the single focus trap, the elastic collision rate (which determines the speed of evaporation) is too low to implement significant cooling before excess loss from background vacuum collisions. For the standing wave, this is not a problem, but with only 4000 atoms initially trapped in each microtrap the phase space density only increases by a factor of $\sim 15$ before running out of atomsthis is similar to the early evaporative cooling results reported in [9]. 
The key to the cross geometry is that it provides a relatively large loading volume $(\sim 2 \mathrm{x}$ greater than the single focus trap) as well as tight confinement in three dimensions, provided the atoms are localized at the intersection region (the 'dimple'). Our current understanding of the system is that it is not the laser cooling process alone that provides this localization (and corresponding high densities), but rather this results in large part from a rapid thermalization and evaporation of the atoms in the trap following a non-equilibrium loading process from the MOT.

The basic idea is very simple. To a reasonable approximation, sub-Doppler laser cooling provides a density-limited sample of low kinetic energy atoms. Hence we can imagine that when the laser cooling operates in conjunction with the optical dipole potential, it serves to 'fill' the trap with a quasi-uniform density of atoms with low kinetic energy. When the cooling light is extinguished, the initial distribution then relaxes to an approximate Maxwell-Boltzmann distribution appropriate to the trapping potential through a combination of rethermalization and evaporation, and this relaxation process can raise the density significantly, particularly in strongly deformed traps like the cross trap.

We have performed two simple experiments to verify this mechanism. First, instead of laser cooling the atoms into the optical trap, the optical trap is snapped on suddenly after all cooling light has been extinguished, thereby eliminating the role of the laser cooling in trap loading process altogether. Despite the excess energy added to the atoms from the potential energy of the trap, within $100 \mathrm{~ms}$, the optically trapped atoms achieve spatial densities 200 times larger than in the MOT with a phase-space density increase of a factor of 30.

In a second experiment, the horizontal single beam trap is first loaded using the standard methods to achieve the properties in the first column of Table 1. Then the diagonal cross-beam was suddenly switched on. The atoms quickly ( $20 \mathrm{~ms})$ coalesce to the dimple region of the cross with final properties within a factor of 2 of the usual cross trap. As described below, a variation of this method can be used to greatly enhance the number of atoms loaded into the microtraps of the 1-D lattice.

\section{Condensates in large period optical lattices}

Optical lattices have found several important applications in the manipulation of BECs due to the potential of engineering controlled coupling between arrays of condensates. A 1-D lattice was used in the first demonstration of Josephson tunneling between lattice wells [16], the generation of number squeezed states [17], demonstration of dynamical tunneling [18], and in transport measurements [19]. In a series of recent experiment with a 3-D lattice, the Munich group has demonstrated a Mott insulator transition [20], and revivals of the condensate wavefunction [21].

In all of these experiments, a sub-micron wavelength optical lattice is employed, and hence the individual lattice sites are not observed. Instead, the coupling between lattice sites is observed as an interference pattern between many phase coherent condensates. The $\mathrm{CO}_{2}$ laser trap provides a lattice in a much different regime, with a lattice spacing sufficiently large $(5.3 \mu \mathrm{m})$ to optically resolve the individual microtraps [22]. This provides a compelling system to pursue direct observation of 
tunneling phenomena. As we discuss below, it is even possible to isolate only 2 microtraps in the lattice to realize a double well potential.

Direct loading of the 1-D lattice trap yields only 4000 atoms per microtrap, which are too few to evaporatively cool to BEC transition. Our new understanding of the loading process has allowed us to increase the number of atoms trapped in a lattice site by over a factor of 200 , and with this, we have succeeded in creating condensates directly in the lattice trap. To increase the number of atoms loaded in the microtraps, the standing wave trap was intersected with a crossed traveling beam during the loading time, which served to 'funnel' a large number of atoms $\left(\sim 10^{6}\right)$ into only a few microtraps. From these initial conditions, evaporation proceeds readily as with the cross trap, and the BEC transition occurs at a critical temperature, $T_{\mathrm{c}}=800 \mathrm{nK}$ with 110,000 atoms and trap oscillation frequencies of $f_{1}, f_{2}, f_{3}=$ $100,140,3200 \mathrm{~Hz}$.
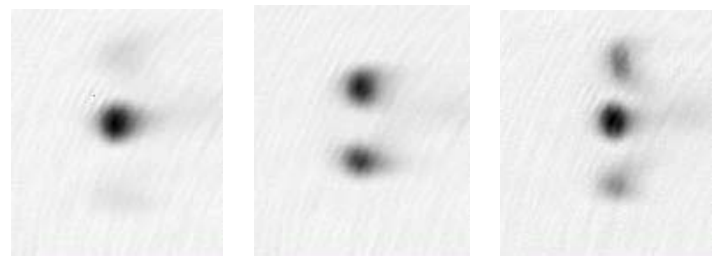

Figure 2: Image of the distinct microtraps following 'magnification' provide by additional harmonic confinement. Images show population of 1, 2 and 3 lattice sites (from left to right).

A unique feature of $\mathrm{CO}_{2}$ lattice is the large, $(5.3 \mu \mathrm{m})$ spacing of the lattice sites. Our current imaging system lacks sufficient resolution to image the individual microtraps. While this will be upgraded in the future, in the mean time, we have developed a technique to measure the site occupancy in the standing wave traps by converting the spatial separation to a separation in momentum space during time-offlight imaging. This is done by superposing a large-scale optical harmonic potential along the direction of the standing wave, and releasing the microtraps into this potential for a short duration before final release of the atoms. Each microtrap is initially located at a different elevation of the added potential and is subsequently accelerated towards the bottom of the potential at different rates. When the added potential is also turned off, the microtrapped atoms will separate in space according to their final velocity. Images acquired in this fashion are shown in Figure 2. By varying the relative strengths of the optical beams, we can have reasonably precise control over the occupation of the microtraps. We anticipate that this will be a convenient experimental technique for studying the dynamics and interplay of these microtrap condensate arrays. Finally, we hope to be able to use the added potential to energetically bias two of the microtraps, thereby isolating them from the adjacent microtraps. This type of potential tailoring should allow us to pursue studies of Josephson type oscillations between adjacent condensates.

One of the hallmarks of BEC is the macroscopic quantum coherence of the gas. In the well-known experiment performed by the MIT group [23], this coherence was 
observed as matter-wave interference by releasing two condensates trapped in an elongated magnetic trap separated by an optical dipole barrier. We have recently been able to observe interference fringes in time of flight images of our released condensates as shown in Figure 3.
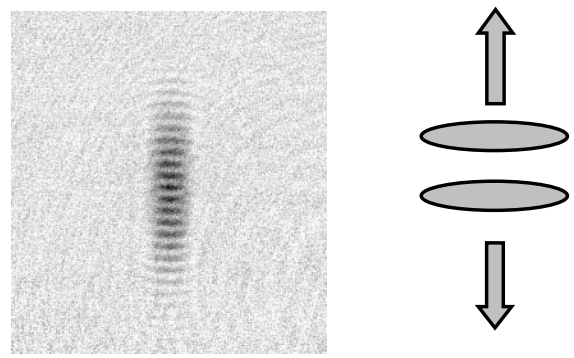

Figure 3: Matter wave interference fringes between 2 condensates localized in separate microtraps. The geometry of microtraps is shown on the right, and the arrows indicate the direction of the fastest expansion.

The fringe spacing resulting from two expanding condensates is given by the de Broglie wavelength of the relative motion of the two independent condensates $\lambda_{d B}=h / m v_{r}$, where $v_{r}=d / \tau$ is determined solely by the separation of the condensates, $d$, and the time, $\tau$, that the condensates are allowed to freely expand before imaging [23]. For our experiment, the spacing of the condensates is $5.3 \mu \mathrm{m}$ (fixed by the standing wave), and the expansion time is $15 \mathrm{~ms}$; this yields a relative velocity of $380 \mu \mathrm{m} / \mathrm{s}$ and a fringe spacing of $12.3 \mu \mathrm{m}$.

\section{Ferromagnetic Spinor condensates}

Bose-Einstein condensates of atoms with spin degrees of freedom offer an entirely new form of coherent matter with complex internal quantum structures. While most current experiments employ magnetic trapping techniques in which the spin degree of freedom is fixed, investigations of multi-component condensates have been explored by utilizing two coupled hyperfine states in ${ }^{87} \mathrm{Rb}[24,25]$, and by $F=$ 1 spinor condensates of sodium by transferring spin polarized BEC made in magnetic traps into far-off resonant optical traps [8]. The MIT group subsequently studied the ground state properties of the spinor condensates and observed ground state domain structures [26], metastable domains [27], and quantum spin tunneling [28].

Depending on the scattering lengths in different angular momentum channels, $F$ $=1$ spinor condensates can be either ferromagnetic or anti-ferromagnetic [29], and the corresponding ground state structure and dynamical properties of the two cases are very distinct. While the sodium spinor is anti-ferromagnetic, the ${ }^{87} \mathrm{Rb} F=1$ spinor is ferromagnetic in nature $[30,31]$. Hence, our all-optical route to BEC 
provides an excellent opportunity for initial exploration of ferromagnetic spinor quantum gases.

To measure the spinor condensates, we perform time-of-flight expansion in a Stern-Gerlach type gradient magnetic field. A typical result is shown in Figure 4 for clouds at different temperatures. As evidenced by the images, the different spin components condense at different temperatures, with the $m_{F}=-1$ component condensing first, followed by the $m_{F}=0$ component and lastly the $m_{F}=1$.
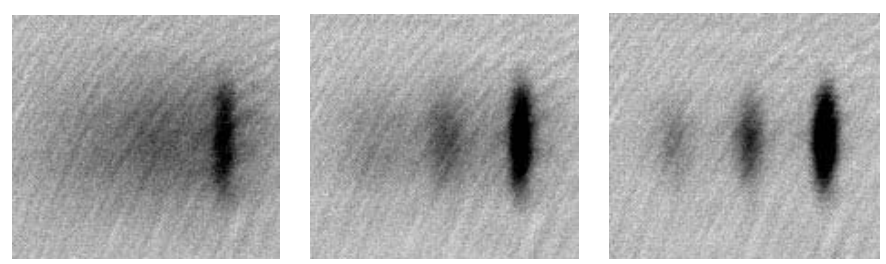

Figure 4: Images of cloud of condensate atoms freely expanding in a Stern-Gerlach field gradient at three different cloud temperatures. The three magnetic spin components $(1,0,-1$, left to right) are spatially separated by the applied gradient.

The different spin components condense at different temperatures.

The origin of the magnetization produced in our all-optical condensates remains to be determined; however we have developed empirical techniques to manipulate the magnetization of the cloud. By applying different magnetic field gradients during the evaporation process, we can significantly alter the spin population (see Figure 5). This capability provides a good starting point for further investigations of the ferromagnetic spinor ground state wavefunctions as well as the associated dynamics of spin mixing which was considered theoretically in [32].
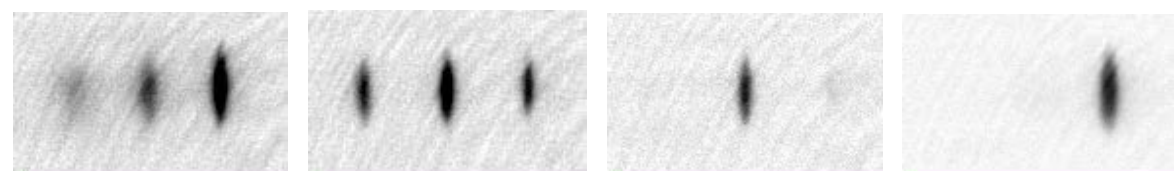

Figure 5: Images showing control the condensate magnetization. Left to right: standard condensate, mixed state, pure $m_{F}=0$ state, pure $m_{F}=-1$ state.

\section{Conclusion and Acknowledgements}

In summary, we have demonstrated an all-optical approach to BEC. It offers simplicity and an order-of-magnitude increase in speed compared to traditional approaches. We have extended the technique to a large-period lattice geometry and observed interference between two independently trapped condensates in the lattice. Finally, we have begun exploration into the spinor nature of the condensates naturally formed in our traps. 
We would like to thank Sally Maddocks and David Zhu for their technical assistance in this work. We gratefully acknowledge support from ARDA and DARPA.

\section{References}

1. Barrett, M.D., J.A. Sauer, and M.S. Chapman, Phys Rev Lett, 87, 010404 (2001).

2. $\quad$ Anderson, M.H., et al., Science, 269, 198 (1995).

3. $\quad$ Davis, K.B., et al., Phys Rev Lett, 75, 3969 (1995).

4. Bradley, C.C., C.A. Sackett, and R.G. Hulet, Phys Rev Lett, 78, 985 (1997).

5. Han, D.J., M.T. DePue, and D.S. Weiss, Phys Rev A, 6302, 023405 (2001).

6. Ido, T., Y. Isoya, and H. Katori, Phys Rev A, 6106, 061403 (2000).

7. Stamper-Kurn, D.M., et al., Phys Rev Lett, 81, 2194 (1998).

8. Stamper-Kurn, D.M., et al., Phys Rev Lett, 80, 2027 (1998).

9. Adams, C.S., et al., Phys Rev Lett, 74, 3577 (1995).

10. Savard, T.A., K.M. OHara, and J.E. Thomas, Phys Rev A, 56, R1095 (1997).

11. Gehm, M.E., et al., Physical Review A, 58, 3914 (1998).

12. Takekoshi, T. and R.J. Knize, Opt Lett, 21, 77 (1996).

13. Friebel, S., et al., Appl Phys B-Lasers O, 67, 699 (1998).

14. Kuppens, S.J.M., et al., Phys Rev A, 6201, 3406 (2000).

15. O'Hara, K.M., et al., Phys Rev A, 6304, 3403 (2001).

16. Anderson, B.P. and M.A. Kasevich, Science, 282, 1686 (1998).

17. Orzel, C., et al., Science, 291, 2386 (2001).

18. Hensinger, W.K., et al., Nature, 412, 52 (2001).

19. Cataliotti, F.S., et al., Science, 293, 843 (2001).

20. Greiner, M., et al., Nature, 415, 39 (2002).

21. Greiner, M., et al., Nature, 419, 51 (2002).

22. Scheunemann, R., et al., Phys Rev A, 6205, 1801 (2000).

23. Andrews, M.R., et al., Science, 275, 637 (1997).

24. Matthews, M.R., et al., Phys Rev Lett, 83, 2498 (1999).

25. Williams, J.E. and M.J. Holland, Nature, 401, 568 (1999).

26. Stenger, J., et al., Nature, 396, 345 (1998).

27. Miesner, H.J., et al., Phys Rev Lett, 82, 2228 (1999).

28. Stamper-Kurn, D.M., et al., Phys Rev Lett, 83, 661 (1999).

29. Ho, T.L., Phys Rev Lett, 81, 742 (1998).

30. Klausen, N.N., J.L. Bohn, and C.H. Greene, Phys Rev A, 6405, 053602 (2001).

31. van Kempen, E.G.M., et al., Phys Rev Lett, 88, 093201 (2002).

32. Law, C.K., H. Pu, and N.P. Bigelow, Phys Rev Lett, 81, 5257 (1998). 
